CLINICAL STUDY

\title{
Psychometric performance of the CushingQoL questionnaire in conditions of real clinical practice
}

\author{
Alicia Santos, Eugenia Resmini, Mª Antonia Martínez-Momblán, Iris Crespo, Elena Valassi, Montserrat Roset ${ }^{1}$, \\ Xavier Badia ${ }^{1}$ and Susan M Webb \\ Endocrinology/Medicine Departments, Hospital de Sant Pau, IIB-Sant Pau, Centro de Investigación Biomédica en Red de Enfermedades Raras (CIBERER \\ Unit 747), ISCIII, Universitat Autonoma de Barcelona (UAB), C/Sant Antoni Maria Claret 167, 08025 Barcelona, Spain and ${ }^{1}$ Health Economics and \\ Outcomes Research, IMS Health, and Centro de Investigación Biomédica en Red en Enfermedades Raras (CIBERER Unit 747), ISCIII, Barcelona, Spain \\ (Correspondence should be addressed to S M Webb; Email: swebb@santpau.cat)
}

\begin{abstract}
Objective: To evaluate health-related quality of life (HRQoL) in Cushing's syndrome (CS) with the disease-generated CushingQoL questionnaire and to confirm its psychometric properties of test-retest reliability and sensitivity to change.

Design: Clinical practice conditions in a tertiary referral center.

Methods: The CushingQoL and EuroQoL questionnaires were administered at baseline and during follow-up and correlated with clinical parameters in 59 patients with CS. To check test-retest reliability, stable patients (either biochemically cured or with active hypercortisolism) were evaluated twice. To investigate sensitivity to change, new patients were evaluated at diagnosis and twice more following improvement after successful surgery.

Results: At baseline, patients with active disease scored lower (indicating worse HRQoL) than those cured on the CushingQoL $(46 \pm 14$ vs $58 \pm 20, P<0.05)$ but not on the EuroQoL-visual analog scale (VAS; $64 \pm 20$ vs $70 \pm 16, P$ NS). Test-retest reliability of CushingQoL was confirmed in stable patients, both in the 'cured group' (intraclass correlation coefficient (ICC) $=0.78, n=34$ ) and in the 'active group' (ICC $=0.66, n=14)$. Sensitivity to change was confirmed in the 'improvement group' $(n=11)$, as the CushingQoL score increased $4 \pm 1.5$ and $9 \pm 3$ months after surgery $(P<0.01$ and $<0.001$ respectively); the EuroQoL-VAS only improved after $9 \pm 3$ months $(P<0.01)$. Effect sizes were 1.02 and 1.86 for CushingQoL at $4 \pm 1.5$ and $9 \pm 3$ months respectively. Finally, scores of both questionnaires were correlated $(r=0.504 ; P<0.001)$.

Conclusions: The CushingQoL questionnaire shows good test-retest reliability and sensitivity to change in clinical practice conditions.
\end{abstract}

European Journal of Endocrinology 167 337-342

\section{Introduction}

Cushing's syndrome (CS) occurs after chronic exposure to an excess of glucocorticoids (GCs). When endogenous, the cause is usually an adenoma located in the pituitary (causing overproduction of ACTH and known as Cushing's disease), in the adrenals, or more rarely in any other part of the body (ectopic secretion of ACTH by a tumor). Adrenal carcinomas are another rarer cause of CS $(1,2)$.

Chronic exposure to GC may produce central obesity, facial plethora (moon face), red striae, thin skin, hypertension, osteoporosis, fatigue, insomnia, depression, anxiety, cognitive problems, lack of libido, and poor health-related quality of life (HRQoL). Although it was initially thought that impairment was only present when the disease was active, recent evidence confirms persistent impairment of HRQoL, even years after biochemical cure $(3,4,5)$.

HRQoL in CS was shown to be impaired with generic questionnaires as the SF-36, the World Health Organization Quality of Life Scale-abbreviated version (WHOQOL-BREF), or the General Health Questionnaire of 28 and 12 items (GHQ-28, GHQ-12) (3, 5, 6, 7, 8, 9, $10,11)$. However, these questionnaires often do not assess specific problems of the disease. Furthermore, as generic questionnaires, they may be less sensitive to clinical changes. Recently, the CushingQoL questionnaire was designed specifically for patients with CS; it initially demonstrated to be feasible, reliable, and valid (12). This study evaluates the psychometric properties of test-retest reliability and sensitivity to change of the CushingQoL questionnaire in clinical practice conditions. 


\section{Materials and methods}

\section{Patients}

Patients were approached during clinical follow-up visits in the Hospital de Sant Pau between October 2007 and December 2011. The Ethics Committee of the Hospital approved the study; after obtaining the informed consent, patients were asked to complete the CushingQoL questionnaire and the generic EuroQoL questionnaire (13). Patients were selected if they had completed the questionnaires at least twice during the follow-up at preselected intervals.

To evaluate test-retest reliability, the questionnaires were administrated twice to cured patients ("cured group') and to patients with active hypercortisolism ('active group') to guarantee its use in both populations. Patients were considered biochemically cured after surgery if adrenal insufficiency was demonstrated or if morning cortisol suppression $(<50 \mathrm{nmol} / \mathrm{l})$ was observed after $1 \mathrm{mg}$ dexamethasone overnight (14) and if repeated 24-h urinary free cortisol measures were normal $(<280 \mathrm{nmol} / \mathrm{l})$. The time between both administrations was $9 \pm 3$ months for the 'cured group' and $3 \pm 1.5$ months for the 'active group'.

To evaluate sensitivity to change, patients with active hypercortisolism were investigated at baseline when CS was diagnosed and again $4 \pm 1.5$ and $9 \pm 3$ months after successful surgery ('improvement group').

\section{Questionnaires}

EuroQoL is a generic, self-administered questionnaire of HRQoL where the patient is asked to evaluate current health. It has been used to investigate multiple diseases as it is short and simple (15). In the first part, the patient has to evaluate the level of impairment of five dimensions (EuroQoL-5D profile): mobility, self-care, usual activities, pain/discomfort, and anxiety/depression. Each dimension is evaluated as a single item with three possible answers (no impairment, some impairment, and severe impairment). Patients were classified as presenting impairment (some impairment or severe impairment) or no impairment. The second part of the questionnaire consists of a visual analog scale (VAS) similar to a thermometer that goes from 0 to 100 and represents the perceived health status of the patient on that day (EuroQoL-VAS). Patients mark the scale at the point they currently perceive their health, higher scores indicating better QoL.

CushingQoL is a disease-generated, unidimensional, self-administered questionnaire, which consists of 12 items referring to problems relevant to patients with CS, with five categories of response to each item on a Likert scale (related to frequency and degree of agreement with the sentence) (12). The time frame of the items refers to the previous 4 weeks. Total score is obtained by adding all items (scored from 1 to 5 ) and therefore ranges from 12 to 60 . Scores are subsequently transformed to a scale from 0 (score 12) to 100 (score 60). Higher values correspond to better QoL.

\section{Statistical analysis}

Analysis was performed using SPSS 18.0 statistical package for Windows (SPSS, Inc., Chicago, IL, USA). Data distribution was analyzed by the KolmogorovSmirnov test. Quantitative data are expressed as mean and s.D. (Gaussian distribution). Comparisons of continuous variables between two groups were performed using Student's $t$-test and comparisons of categorical variables were performed using the $\chi^{2}$ test. Correlations between continuous variables were analyzed with Pearson's correlation coefficient. In all the comparisons, a $\mathrm{P}<0.05$ was considered statistically significant. Test-retest reliability of the CushingQoL questionnaire was analyzed by the intraclass correlation coefficient (ICC) in both the 'active' and 'cured' groups. Sensitivity to change was evaluated with the effect size of the global score in the improvement group'. ANOVA for repeated measures followed by a simple contrast was also used. The effect size was defined as the difference in the mean score between baseline and follow-up divided by the baseline s.D.

\section{Results}

All patients who were approached agreed to participate $(n=59,50$ females, age $48.3 \pm 12.4$ years). Forty-seven suffered with CS of pituitary origin, ten due to an adrenal adenoma, one ectopic ACTH secretion of unknown origin, and one due to ACTH-independent macronodular hyperplasia (AIMAH; Table 1). Forty-two had undergone surgery (36 pituitary surgery and six

Table 1 Baseline clinical characteristics of patients included in the study.

\begin{tabular}{|c|c|c|c|}
\hline & $\begin{array}{l}\text { 'Cured } \\
\text { group' }\end{array}$ & $\begin{array}{l}\text { 'Active } \\
\text { group' }\end{array}$ & $\begin{array}{l}\text { 'Improvement } \\
\text { group' }\end{array}$ \\
\hline Number of patients & 34 & 14 & 11 \\
\hline Gender (M/F) & $6 / 28$ & $3 / 11$ & $0 / 11$ \\
\hline Age (mean \pm s.D.) & $49.9 \pm 12.2$ & $50.4 \pm 11.5$ & $41.2 \pm 12.7$ \\
\hline \multicolumn{4}{|l|}{$\begin{array}{l}\text { Origin of Cushing's } \\
\text { syndrome }\end{array}$} \\
\hline Pituitary & 28 & 12 & 7 \\
\hline Adrenal & 6 & 0 & 4 \\
\hline $\begin{array}{l}\text { Ectopic ACTH } \\
\text { secretion }\end{array}$ & 0 & 1 & 0 \\
\hline AIMAH & 0 & 1 & 0 \\
\hline $\begin{array}{l}\text { Surgery } \\
\text { (pituitary/adrenal) }\end{array}$ & 34 & 8 & 0 \\
\hline Radiotherapy & 9 & 6 & 0 \\
\hline
\end{tabular}

'Cured group', patients were stable at both evaluations, fulfilling cure criteria. 'Active group', patients were stable at both evaluations, with active hypercortisolism. 'Improvement group', active hypercortisolism at baseline, cured at reevaluation. $M$, males; F, females; AIMAH, ACTH-independent macronodular adrenal hyperplasia. 


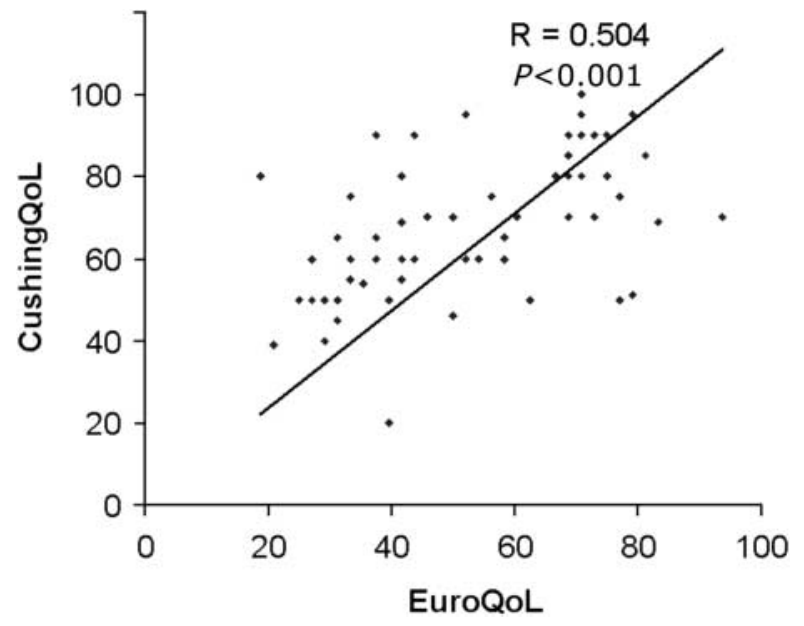

Figure 1 Correlation between all basal CushingQoL and the EuroQoL-VAS scores included in the study $(n=59)$.

adrenal surgery), 15 had received radiotherapy, 11 were taking hydrocortisone for postoperative adrenal insufficiency, 13 were taking steroidogenesis inhibitors at the moment of the study (five on ketoconazole, three on metyrapone, and five on both), one was taking cabergoline, and another losartan (a male with AIMAH found to respond to angiotensin receptor antagonists).

The 'cured group' comprised 34 patients biochemically cured and clinically stable throughout the study period. The 'active group' included 14 patients with active hypercortisolism that did not fulfill cure criteria at any time point when the questionnaires were administered (ten patients taking ketoconazole and/or metyrapone, one on losartan, and one on cabergoline). The 'improvement group' included 11 patients seen at diagnosis who later underwent surgery that cured their hypercortisolism. Details of patients in each group are presented in Table 1; there were no differences in age between the groups.

\section{Comparison of patients with active and cured hypercortisolism}

All basal scores corresponding to patients with active hypercortisolism ('active group' and basal score of the 'improvement group') were compared with those with cured disease ('cured group'). CushingQoL scores were worse in patients with active disease than in those cured $(46 \pm 15$ vs $58 \pm 20, P<0.05)$. No statistically significant differences were seen in the EuroQoL-VAS $(64 \pm 20$ in active vs $70 \pm 16$ in cured disease, $P$ NS). Considering all basal evaluations, scores of both questionnaires were correlated $(r=0.504 ; P<0.001$; Fig. 1).

No difference in frequency of impairments of the EuroQoL-5D profile was found between patients with active and cured hypercortisolism (mobility $48 \%$ in active vs $32 \%$ in cured patients; self-care, 16 vs $6 \%$; usual activities, 52 vs $32 \%$; anxiety/depression, 64 vs $56 \%$; and pain/discomfort, 56 vs $65 \%$; Fig. 2 ).

\section{Other clinical features}

No differences were seen for the EuroQoL-VAS or CushingQoL score between males $(n=9$, three with active disease) and females $(n=50,22$ with active disease), or between patients with hypercortisolism of adrenal ( $n=10$, four with active disease) and pituitary origin ( $n=47,19$ with active disease), although there was a trend for lower CushingQoL scores in patients of adrenal origin $(P=0.073)$. No differences were found between patients who had undergone radiotherapy ( $n=15$, six with active disease) or not $(n=44,19$ with active disease). No effects on the EuroQoL-VAS or CushingQoL score were seen when cured patients who required hydrocortisone for postoperative adrenal insufficiency $(n=11)$ were compared with those cured patients who did not $(n=23)$, or in patients with active hypercortisolism treated with steroidogenesis-inhibiting drugs $(n=13)$ or not $(n=12)$. Finally, no correlation was found for CushingQoL or EuroQoL-VAS and age in the 59 patients $(r=-0.040, P=\mathrm{NS} ; r=-0.214$, $P=$ NS respectively).

\section{Test-retest reliability}

'Cured group' No changes were observed in either questionnaire when first evaluation was compared with the second evaluation $9 \pm 3$ months later (EuroQoLVAS, $70 \pm 16$ and $70 \pm 13$; CushingQoL, $58 \pm 21$ and $57 \pm 20$ ). The ICC for CushingQoL was 0.78 $(P<0.001)$, indicating an excellent test-retest reliability.

'Active group' No changes were observed either for the scores of the questionnaires in patients with active

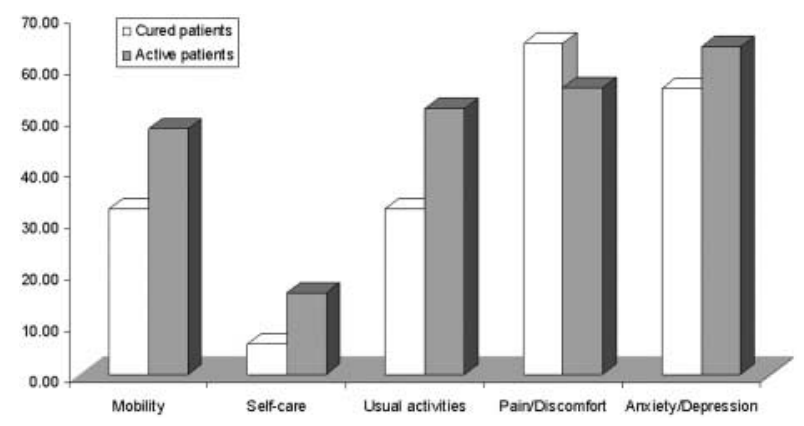

Figure 2 Percentage of patients referring problems in the EuroQoL dimensions (EuroQoL-5D profile), comparing cured ('cured group', $n=34$ ) and active patients ('active group', $n=14$, plus 'improvement group', $n=11$ ). 


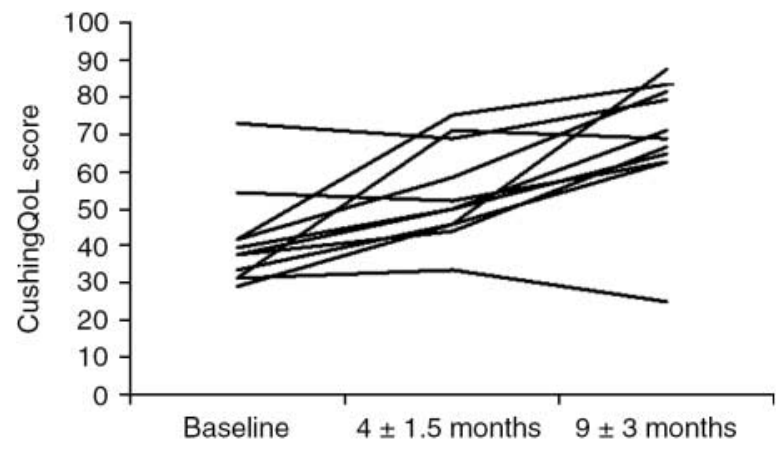

Figure 3 Individual CushingQoL scores at baseline and after surgery in the 'improvement group'.

disease, evaluated at baseline and $3 \pm 1.5$ months later (EuroQoL-VAS, $66 \pm 21$ and 57 \pm 23 ; CushingQoL, $49 \pm 15$ and $47 \pm 17)$. The ICC of $0.66(P<0.001)$ obtained in this group was slightly lower and indicates moderate to good reliability.

\section{Sensitivity to change ('improvement group')}

When presurgery baseline data were compared with follow-up evaluations after endocrine control of hypercortisolism, improvement in the EuroQoL-VAS score was observed at $9 \pm 3$ months $(P<0.01)$, but not at $4 \pm 1.5$ months (Table 2 ). The CushingQoL score improved earlier at $4 \pm 1.5$ months $(P<0.01)$ and remained significant $9 \pm 3$ months postsurgery $(P<0.001$; Fig. 3). Effect sizes were 1.02 and 1.86 for CushingQoL at 4 and 9 months respectively. Assuming that an effect size of 0.8 or larger corresponds to a large change, the improvement in CushingQoL score demonstrates the sensitivity to change of the questionnaire. Even though a moderate correlation (Pearson's correlation coefficient of 0.37) was found between baseline and final CushingQoL scores in these 11 patients, we could not confirm that a worse baseline score was associated with a greater improvement after surgery.

\section{Discussion}

The psychometric properties of test-retest reliability and sensitivity to change of the CushingQoL questionnaire have been evaluated in clinical practice conditions. Stability of scores over time (test-retest reliability) has been shown in clinically stable patients, both cured and active. Furthermore, a good correlation of the scores of the questionnaire at first and second evaluations was found (ICC $=0.66$ for cured patients and $I C C=0.78$ for active patients). A shorter interval was chosen for reevaluation in the 'active group' $(3 \pm 1.5$ vs $9 \pm 3$ months) as the aim of treatment is to normalize cortisol in the shortest time possible, so that few patients remained active after 5-6 months of medical therapy.
Sensitivity to change has also been confirmed in CS patients who underwent successful surgery and who were followed over the subsequent year at different time points. In these patients, improvement was demonstrated earlier with the CushingQoL questionnaire than with the EuroQoL-VAS. This suggests that the items included in the specific CushingQoL questionnaire are more sensitive than those of the generic questionnaire in detecting perceived HRQoL, as has been observed in pituitary diseases for which disease-generated questionnaires are available $(15,16)$.

As expected, patients with active hypercortisolism scored worse on the CushingQoL questionnaire than biochemically cured patients. Similar results were found in recent studies using CushingQoL, with a mean score of $39 \pm 17$ for active patients and $52 \pm 18$ for cured patients $(17,18)$. Nevertheless, up to two-thirds of cured patients still have limitations in the dimensions evaluated by the EuroQoL-5D profile, especially for pain/discomfort, which tends to be higher in surgically cured patients $(65 \%)$ than in active disease $(56 \%)$. These data confirm that biochemical cure of hypercortisolism after treatment for CS is not associated with complete normalization of HRQoL. In fact, HRQoL in cured CS patients has been reported to be worse than in normal population and in other pituitary tumors, using several generic questionnaires $(3,4,5,19)$. One study found that HRQoL was impaired in all items, except pain (measured by SF-36) and sleep (Nottingham Health Profile questionnaire) in cured CS patients compared with controls (4). Other data demonstrate that HRQoL measured by SF-36 improved but was still impaired after transsphenoidal surgery when compared with reference values (3). Furthermore, improvement after surgery has been shown not to be immediate. Fatigability in some patients after bilateral adrenalectomy may take several months or longer to resolve (9); indeed, fatigability was still reported in $46 \%$ of the patients 1 year after surgery (19).

No differences between males and females for either the CushingQoL or EuroQoL-VAS scores were found, although as expected in CS, there was a clear female preponderance ( $85 \%$ of the patients). In general, HRQoL is usually more impaired in women than in men. But reports in patients with CS are conflicting; while in a cohort of 58 patients with cured Cushing's disease, women did show worse scores than men in one of

Table 2 Sensitivity to change of the CushingQoL and EuroQoLVAS questionnaires in patients who were biochemically cured of hypercortisolism after surgery for Cushing's syndrome.

\begin{tabular}{lccc}
\hline & Baseline & $\begin{array}{c}\mathbf{4} \pm \mathbf{1 . 5} \text { months } \\
\text { postsurgery }\end{array}$ & $\begin{array}{c}\mathbf{9} \pm \mathbf{3} \text { months } \\
\text { postsurgery }\end{array}$ \\
\hline CushingQoL & $41 \pm 15$ & $54 \pm 13^{\star}$ & $68 \pm 17^{\dagger}$ \\
EuroQoL-VAS & $62 \pm 20$ & $69 \pm 17$ & $78 \pm 17^{\star}$
\end{tabular}

VAS, visual analog scale. ${ }^{\star} P<0.01$ and ${ }^{\dagger} P<0.001$ vs baseline. 
the dimensions of the generic Nottingham Health Profile (4), another recent study which reported that baseline CushingQoL and EuroQoL scores in active CS patients from a large European database found no gender difference (17). We found no correlations between the questionnaires and age, or differences related to prior radiotherapy treatment, in accordance with a previous report in which 11 of the 58 patients were irradiated (4). Patients with CS of adrenal origin tended to have lower QoL scores than those of pituitary origin, but no significant differences were found, in agreement with a larger European study (17).

Patients who were adrenal insufficient after surgery and required hydrocortisone did not differ in the CushingQoL score and EuroQoL-VAS compared with those who were eucortisolemic. Previous studies have reported no effects of hydrocortisone substitution on QoL in CS $(3,18)$ but also that adrenal insufficiency in general, as well as Addison's disease and hypopituitarism, are related to worse HRQoL $(4,20)$. No effect of cortisol-lowering drugs, like metyrapone and ketoconazole, was observed when comparing patients who were treated or not with these drugs, even though the groups were small. Probably, the great burden of the disease negatively influences QoL to a greater extent than all these other factors. In fact, CS probably determines impaired HRQoL in a multifactorial way, including physical and psychological problems, as well as the necessity to undergo repeated testing and check-ups, and the need for chronic medication.

A limitation of this study was the small sample size especially of the 'active group' and the 'improvement group', which is difficult to avoid in a rare disease as CS followed in a single center. An effect of age, gender, origin, concomitant medications, radiotherapy, or a worse baseline CushingQoL score cannot be ruled out completely and larger multicentric studies would be required to understand the influence of these factors on HRQoL in CS. Furthermore, this study has been carried out in clinical practice conditions, not in a controlled clinical trial with strict time points. However, the employed conditions are closer to real life and therefore reflect clinical practice, which in itself varies depending on different countries and health systems.

In conclusion, the CushingQoL questionnaire appears to be a useful and promising tool for the evaluation of HRQoL in CS patients in clinical practice due to its simplicity, reduced number of items, and ability to detect clinically relevant changes over time. Next to it being feasible, reliable, and valid, we have now demonstrated that the CushingQoL questionnaire has good test-retest reliability and sensitivity to change.

\section{Declaration of interest}

The authors declare that there is no conflict of interest that could be perceived as prejudicing the impartiality of the research reported.

\section{Funding}

Supported in part by a grant from ISCIII of the Spanish Ministry of Science and Innovation (MICINN) FIS080302 and the European Commission (ERCUSYN PHP800200). The CushingQoL questionnaire can be obtained from the corresponding author.

\section{References}

1 Arnaldi G, Angeli A, Atkinson AB, Bertagna X, Cavagnini F, Chrousos GP, Fava GA, Findling JW, Gaillard RC, Grossman AB, Kola B, Lacroix A, Mancini T, Mantero F, Newell-Price J, Nieman LK, Sonino N, Vance ML, Giustina A \& Boscaro M. Diagnosis and complications of Cushing's syndrome, a consensus statement. Journal of Clinical Endocrinology and Metabolism 2003 88 5593-5602. (doi:10.1210/jc.2003-030871)

2 Nieman LK, Biller BM, Findling JW, Newell-Price J, Savage M, Setewart P \& Montori VM. The diagnosis of Cushing's syndrome: an Endocrine Society Clinical Practice Guideline. Journal of Clinical Endocrinology and Metabolism 200893 1526-1540. (doi:10.1210/jc.2008-0125)

3 Lindsay JR, Nansel T, Baid S, Gumowski J \& Nieman LK. Long-term impaired quality of life in Cushing's syndrome despite initial improvement after surgical remission. Journal of Clinical Endocrinology and Metabolism 200691 447-453. (doi:10.1210/jc. 2005-1058)

4 Van Aken MO, Pereira AM, Biermasz NR, van Thiel SW, Hoftijzer HC, Smit JW, Roelfsema F, Lamberts SW \& Romijn JA. Quality of life in patients after long-term biochemical cure of Cushing's disease. Journal of Clinical Endocrinology and Metabolism 200590 3279-3286. (doi:10.1210/jc.2004-1375)

5 Heald AH, Ghosh S, Bray S, Gibson C, Anderson SG, Buckler H \& Fowler HL. Long-term negative impact on quality of life in patients with successfully treated Cushing's disease. Clinical Endocrinology 200461 458-465. (doi:10.1111/j.1365-2265. 2004.02118.x)

6 Hawn MT, Cook D, Deveney C \& Sheppard BC. Quality of life after laparoscopic bilateral adrenalectomy for Cushing's disease. Surgery 2002132 1064-1068. (doi:10.1067/msy. 2002.128482)

7 Johnson MD, Woodburn CJ \& Vance ML. Quality of life in patients with a pituitary adenoma. Pituitary 20036 81-87. (doi:10.1023/ B:PITU.0000004798.27230.ed)

8 Ding XF, Li HZ, Yan WG, Gao Y \& Li XQ. Role of adrenalectomy in recurrent Cushing's disease. Chinese Medical Journal 2010123 $1658-1662$.

9 Smith PW, Turza KC, Carter CO, Vance ML, Laws ER \& Hanks JB. Bilateral adrenalectomy for refractary Cushing's disease: a safe and definitive therapy. Journal of the American College of Surgeons 2009208 1059-1064. (doi:10.1016/j.jamcollsurg. 2009.02.054)

10 Mattoo SK, Bhansali AK, Gupta N, Grover S \& Malhotra R. Psychosocial morbidity in Cushing disease: a study from India. Endocrine 200935 306-311. (doi:10.1007/s12020-009-9182-2)

11 Van der Klaauw AA, Kars M, Biermasz NR, Roelfsema F, Dekkers OM, Corssmit EP, van Aken MO, Havekes B, Pereira AM, Pijl H, Smit JW \& Romijn JA. Disease-specific impairments in quality of life during long-term follow-up of patients with different pituitary adenomas. Clinical Endocrinology 200869 775-784. (doi:10.1111/j.1365-2265.2008.03288.x)

12 Webb SM, Badia X, Barahona MJ, Colao A, Strasburger CJ, Tabarin A, van Aken MO, Pivonello R, Stalla G, Lamberts SW \& Glusman JE. Evaluation of health-related quality of life in patients with Cushing's syndrome with a new questionnaire. European Journal of Endocrinology $2008 \mathbf{1 5 8}$ 623-630. (doi:10.1530/ EJE-07-0762) 
13 Badía X, Roset M, Herdman M \& Kind P. A comparison of United Kingdom and Spanish general population time trade-off values for EQ-5D health states. Medical Decision Making 200121 7-16. (doi:10.1177/0272989X0102100102)

14 Brooks R. EuroQol: the current state of play. Health Policy 199637 53-72. (doi:10.1016/0168-8510(96)00822-6)

15 Woodhouse LJ, Mukherjee A, Shalet SM \& Ezzat S. The influence of growth hormone status on physical impairments, functional limitations, and health-related quality of life in adults. Endocrine Reviews 200627 287-317. (doi:10.1210/er.2004-0022)

16 Miller A, Doll H, David J \& Wass J. Impact of musculoskeletal disease on quality of life in long-standing acromegaly. European Journal of Endocrinology 2008 158 587-593. (doi:10.1530/EJE-07-0838)

17 Valassi E, Santos A, Yaneva M, Toth M, Strasburger CJ, Chanson P, Wass JAH, Chabre O, Pfeifer M, Feelders RA, Tsagarakis S, Trainer PJ, Franz H, Zopf K, Zacharieva S, Lamberts SWJ, Tabarin A \& Webb SM. The European Registry on Cushing's syndrome: 2-year experience. Baseline demographic and clinical characteristics. European Journal of Endocrinology $2011 \mathbf{1 6 5}$ 383-392. (doi:10.1530/EJE-11-0272)
18 Tiemensma J, Kaptein AA, Pereira AM, Smit JW, Romijn JA \& Biermasz NR. Negative illness perceptions are associated with impaired quality of life in patients after long-term remission of Cushing's syndrome. European Journal of Endocrinology 2011165 527-535. (doi:10.1530/EJE-11-0307)

19 Sippel RS, Elaraj DM, Kebebew E, Lindsay S, Tyrrell JB \& Duh QY. Waiting for change: symptom resolution after adrenalectomy for Cushing's syndrome. Surgery $2008 \mathbf{1 4 4}$ 1054-1061. (doi:10.1016/j.surg.2008.08.024)

20 Løvås K, Curran S, Oksnes M, Husebye ES, Huppert FA \& Chatterjee VK. Development of a disease-specific quality of life questionnaire in Addison's disease. Journal of Clinical Endocrinology and Metabolism 2010 95 545-551. (doi:10.1210/jc.2009-1711)

Received 17 April 2012

Revised version received 5 June 2012

Accepted 7 June 2012 